\title{
LIGNANS FROM WOOD OF CALOCEDRUS FORMOSANA
}

\author{
JIM-MIN FANG, MING-YING LIU and YU-SHIA CHENG* \\ Department of Chemistry, National Taiwan University, Taipei, 10764, Taiwan, Republic of China
}

(Received 18 January 1990)

Key Word Index - Calocedrus formosana; Cupressaceae; heartwood; lignans; 7-oxohinokinin; 4',5-dihydroxy-3,3',4trimethoxylign-7-en-9,9'-olide; 4,4'-dihydroxy-3,3',5-trimethoxylign-7-en-9,9'-olide; hinokinin; savinin; calocedrin; matairesinol

\begin{abstract}
Hinokinin, savinin, calocedrin, matairesinol and three new lignans, 7-oxohinokinin, 4',5-dihydroxy-3,3',4trimethoxylign-7-en-9, $9^{\prime}$-olide and 4,4'-dihydroxy-3,3',5-trimethoxylign-7-en-9,9' olide, were isolated from an acetone extract of the wood of Calocedrus formosana. The structures of new lignans were determined by spectral methods.
\end{abstract}

\section{INTRODUCTION}

The constituents of the heartwood of Calocedrus formosana, a member of Cupressaceae indigenous to Taiwan [1], have been extensively investigated in our laboratory [2-7]. In addition to a large quantity of terpenoid acids, such as shonanic, thujic and chaminic acids, the wood also contains tropolones, monoterpenes, naphthalenetype sesquiterpenes, diterpenoid phenols and lignans, viz. (-)-hinokinin (1), (-)-savinin (hibalactone, 2), and (+)calocedrin (3). On continuing a study of the chemical constituents, we have now isolated (-)-matairesinol (4) $[8,9]$ and three new lignans $5-7$ from an acetone extract of the wood of $C$. formosana.

\section{RESULTS AND DISCUSSION}

Compound 5, [M] $]^{+} m / z 368$, showed resonances of 20 carbons in its ${ }^{13} \mathrm{CNMR}$ spectrum. The resonances of $\delta 172.7(s)$ and $190.9(s)$ are ascribed to two carbonyl carbons. The IR absorptions at 1765 and $1660 \mathrm{~cm}^{-1}$ indicate the presence of $\gamma$-butyrolactone and conjugated ketone moieties. By comparison of the ${ }^{1} \mathrm{H}$ and ${ }^{13} \mathrm{CNMR}$ data with those of hinokinin $[3,10]$, the structure of compound 5 is determined to be 7-oxohinokinin. The H-8 resonance appears at $\delta 4.19$ as a doublet $(J=5.7 \mathrm{~Hz})$. On treatment with a strong base $(t-\mathrm{BuOK}, t-\mathrm{BuOH}$, THF) at room temperature for $34 \mathrm{hr}$, no epimerization took place. Thus, this lignan is considered to have the thermodynamically stable $8,8^{\prime}$-trans-configuration. The absolute configuration $\left(8 S, 8^{\prime} R\right)$ of $(-)-7$-oxohinokinin is tentatively assigned by analogy to (-)-hinokinin and other lignan constituents found in the same plant.

Compounds 6 and 7 , both of them showing $[\mathrm{M}]^{+}$at $m / z 386$, must be isomeric compounds because they have similar IR and UV spectra. The presence of a cinnamic acid $\gamma$-lactone moiety is inferred from IR absorptions at 1740 and $1640 \mathrm{~cm}^{-1}$ as well as from UV absorption at $320 \mathrm{~nm}$. In the ${ }^{1} \mathrm{H}$ NMR spectrum, 6 displayed five aromatic protons, two phenolic hydroxyl protons and resonances at $\delta 3.86,3.87$ and 3.95 attributable to three anisole methyl groups. The positions of hydroxyl and methoxy groups are readily deduced by comparison with the literature [10]. Thus, the structure of 6 is assigned as<smiles>[Y]C(=C1C(=O)OC[C@@H]1Cc1ccc2c(c1)OCO2)c1ccc2c(c1)OCO2</smiles>

$1 Y=H, H$

$5 Y=0$<smiles>COc1cc(C[C@@H]2C(=O)OC[C@@H]2Cc2ccc(O)c(OC)c2)ccc1O</smiles>

4<smiles>[R20]c1cc(/C=C2/C(=O)OC[C@H]2Cc2ccc(O)c(OC)c2)cc(OC)c1[R20]</smiles>

$6 R^{1}=M \theta, R^{2}=H$

$7 R^{1}=H, R^{2}=M e$

(7E)-4',5-dihydroxy-3,3',4-trimethoxylign-7-en-9,9'-olide. On the other hand, the ${ }^{1} \mathrm{H}$ and ${ }^{13} \mathrm{C}$ NMR spectra of 7 $\left(\mathrm{C}_{21} \mathrm{H}_{22} \mathrm{O}_{7}\right)$ revealed that it has symmetry on a phenyl ring; there were only 18 peaks visible in the ${ }^{13} \mathrm{CNMR}$ spectrum. The three methoxy groups appeared at two positions, $\delta 55.8$ and 56.3 (two superimposed carbons). The corresponding proton signals occurred at $\delta 3.80(3 \mathrm{H})$ and $3.85(6 \mathrm{H})$ in the ${ }^{1} \mathrm{H}$ NMR spectrum. The aromatic protons $\mathrm{H}-2$ and $\mathrm{H}-6$ had the same chemical shift of $\delta 6.77(s)$. The structure of 7 is thus $(7 E)-4,4^{\prime}$-dihydroxy3,3'-5-trimethoxylign-7-en-9,9'-olide.

\section{EXPERIMENTAL}

Plant material. Calocedrus formosana (Florin) Florin was collected in the campus of the National Taiwan University. A 
voucher specimen is deposited in the herbarium of the Department of Botany. The skinned and air-dried wood $(600 \mathrm{~g})$ from branches $6-8 \mathrm{~cm}$ diam. was selected for study. After extraction $\times 3$ with $\mathrm{Me}_{2} \mathrm{CO}$, the combined extracts were concd in vacuo to give $20 \mathrm{~g}$ of residue. Components were sepd by $\mathrm{CC}$ on silica gel $(230 \mathrm{~g})$ and elution with hexane-EtOAc gradients. Compound 1 was eluted first, followed by $6,7,2,5,4$ and 3 .

7-Oxohinokinin (5). $\mathrm{C}_{20} \mathrm{H}_{16} \mathrm{O}_{7}$, needle-like crystals (from $\left.\mathrm{CHCl}_{3}-\mathrm{MeOH}\right), \mathrm{mp} 109.5-111^{\circ},[\alpha]_{\mathrm{D}}^{25}+10^{\circ}\left(\mathrm{CHCl}_{3} ; c 0.82\right)$. $\lambda_{\max }^{\mathrm{CHCl}_{3}} \mathrm{~nm}(8): 313(7570), 281(8620), 240(8250)$. IR $v_{\max }^{\text {neat }} \mathrm{cm}^{-1}$ : 1765, 1660. EIMS $m / z$ (rel. int.): $368[\mathrm{M}]^{+}(13), 260$ (5), $242(6)$, $233(9), 227(5), 201(9), 178(10), 160(100), 149(68), 135(33), 131$ (69), 121 (40). ${ }^{1} \mathrm{H}$ NMR $\left(\mathrm{CDCl}_{3}, 300 \mathrm{MHz}\right): \delta 2.75(1 \mathrm{H}, d d, J$ $\left.=13.6,8.2 \mathrm{~Hz}, \mathrm{H}-7^{\prime}\right), 2.79\left(1 \mathrm{H}, d d, J=13.6,7.8 \mathrm{~Hz}, \mathrm{H}-7^{\prime}\right), 3.31$ $(1 \mathrm{H}, m), 4.12\left(1 \mathrm{H}, d d, J=9.0,7.0 \mathrm{~Hz}, \mathrm{H}-9^{\prime}\right), 4.19(1 \mathrm{H}, d, J=5.7 \mathrm{~Hz}$ $\mathrm{H}-8), 4.51\left(1 \mathrm{H}, d d, J=9.0,5.3 \mathrm{~Hz}, \mathrm{H}-9^{\prime}\right), 5.93(2 \mathrm{H}, s), 6.05(2 \mathrm{H}, s)$, $6.61\left(1 \mathrm{H}, d d, J=7.8 \mathrm{~Hz}, 1.2 \mathrm{~Hz}, \mathrm{H}-6^{\prime}\right), 6.63(1 \mathrm{H}, d, J=1.2 \mathrm{~Hz}, \mathrm{H}-$ $\left.2^{\prime}\right), 6.72\left(1 \mathrm{H}, d, J=7.8 \mathrm{~Hz}, \mathrm{H}-5^{\prime}\right), 6.82(1 \mathrm{H}, d, J=8.1 \mathrm{~Hz}, \mathrm{H}-5), 7.30$ $(1 \mathrm{H}, d, J=1.7 \mathrm{~Hz}, \mathrm{H}-2), 7.42(1 \mathrm{H}, d d, J=8.1,1.7 \mathrm{~Hz}, \mathrm{H}-6)$. ${ }^{13} \mathrm{CNMR}\left(\mathrm{CDCl}_{3}, 75 \mathrm{MHz}, \mathrm{DEPT}\right): \delta 38.1\left(t, \mathrm{C}-7^{\prime}\right), 41.4\left(d, \mathrm{C}-8^{\prime}\right)$, $53.6(d, \mathrm{C}-8), 71.9\left(t, \mathrm{C}-9^{\prime}\right), 101.1\left(t, \mathrm{OCH}_{2} \mathrm{O}\right), 102.1\left(t, \mathrm{OCH}_{2} \mathrm{O}\right)$, $107.9(d, \mathrm{C}-2), 108.5\left(d, \mathrm{C}-2^{\prime}\right), 108.6(d, \mathrm{C}-5), 109.1\left(d, \mathrm{C}-5^{\prime}\right), 122.0$ $(d, \mathrm{C}-6), 126.2\left(d, \mathrm{C}-6^{\prime}\right), 130.1(s, \mathrm{C}-1), 131.1\left(s, \mathrm{C}-1^{\prime}\right), 146.6\left(s, \mathrm{C}-4^{\prime}\right)$ $148.1\left(s, \mathrm{C}-3^{\prime}\right), 148.3(s, \mathrm{C}-3), 152.6(s, \mathrm{C}-4), 172.7(s, \mathrm{C}-9), 190.9$ $(s, \mathrm{C}-7)$.

(7E)-4',5-Dihydroxy-3,3',5-trimethoxylign-7-en-9,9'-olide (6) $\mathrm{C}_{21} \mathrm{H}_{22} \mathrm{O}_{7}$, needle-like crystals (obtained by HPLC), mp $130-131^{\circ},[\alpha]_{\mathrm{D}}^{25}-94^{\circ}\left(\mathrm{CHCl}_{3} ; c 0.15\right) . \mathrm{UV} \lambda_{\max } \mathrm{nm}(\varepsilon): 322$ (21620), 299 (17300), 280 (21 700), $238(27410)$. IR $v_{\max } \mathrm{cm}^{-1}$. $3440,1735,1640,1600,1510$. MS $m / z$ (rel. int.): $386\left[\mathrm{M}^{+}{ }^{+}(8), 249\right.$ (23), 205 (3), 193 (7), 189 (7), 161 (12), 137 (100), 122 (23), 105 (6). ${ }^{1} \mathrm{H}$ NMR $\left(\mathrm{CDCl}_{3}\right): \delta 2.57(1 \mathrm{H}, d d, J=14.1,10.2 \mathrm{~Hz}, \mathrm{H}-7$ '), 3.02 $\left(1 \mathrm{H}, d d, J=14.1,3.9 \mathrm{~Hz}, \mathrm{H}-7^{\prime}\right), 3.82\left(1 \mathrm{H}, m, \mathrm{H}-8^{\prime}\right), 3.86(3 \mathrm{H}, s$, $\mathrm{OMe}$ ), 3.87 (3H, s, OMe), 3.95 (3H, $s, \mathrm{OMe}), 4.25$ (2H, $\left.m, \mathrm{H}-9^{\prime}\right)$, $5.50(1 \mathrm{H}, s, \mathrm{OH}), 5.86(1 \mathrm{H}, s, \mathrm{OH}), 6.61(1 \mathrm{H}, d, J=1.8 \mathrm{~Hz}, \mathrm{H}-6)$, $6.69\left(1 \mathrm{H}, b r d, J=8.4 \mathrm{~Hz}, \mathrm{H}-6^{\prime}\right), 6.70\left(1 \mathrm{H}, b r s, \mathrm{H}-2^{\prime}\right), 6.83(1 \mathrm{H}, d, J$ $\left.=8.4 \mathrm{~Hz}, \mathrm{H}-5^{\prime}\right), 6.95(1 \mathrm{H}, d, J=1.8 \mathrm{~Hz}, \mathrm{H}-2), 7.45(1 \mathrm{H}, d, J$ $=1.8 \mathrm{~Hz}, \mathrm{H}-7) \cdot{ }^{13} \mathrm{C} \mathrm{NMR}\left(\mathrm{CDCl}_{3}, \mathrm{DEPT}\right) \delta 37.6\left(t, \mathrm{C}-7^{\prime}\right), 40.1(d$, $\left.\mathrm{C}-8^{\prime}\right), 55.9(q, \mathrm{OMe}), 56.0(q, \mathrm{OMe}), 61.1(q, \mathrm{OMe}), 70.0\left(t, \mathrm{C}-9^{\prime}\right)$, $107.6(d, \mathrm{C}-2), 108.7(d, \mathrm{C}-6), 111.4\left(d, \mathrm{C}-2^{\prime}\right), 114.5\left(d, \mathrm{C}-5^{\prime}\right), 121.5$ $\left(d, \mathrm{C}-6^{\prime}\right), 127.7(s, \mathrm{C}-1), 129.8\left(s, \mathrm{C}-1^{\prime}\right.$ and $\left.\mathrm{C}-8\right), 137.0(s, \mathrm{C}-4), 137.1$ $(d, \mathrm{C}-7), 144.6\left(s, \mathrm{C}-4^{\prime}\right), 146.7\left(s, \mathrm{C}-3^{\prime}\right), 149.5(s, \mathrm{C}-5), 152.3(s, \mathrm{C}-3)$, $172.5(s, \mathrm{C}-9)$.

(7E)-4,4'-Dihydroxy-3,3',5-trimethoxylign-7-en-9,9'-olide (7). $\mathrm{C}_{21} \mathrm{H}_{22} \mathrm{O}_{7}$, needle-like crystals (obtained by HPLC), mp $112-113^{\circ},[\alpha]_{\mathrm{D}}^{25}-16.3^{\circ}\left(\mathrm{CHCl}_{3} ; c 0.3\right)$. UV $\lambda_{\max } \mathrm{nm}(\varepsilon): 320$ (17000), 299 (16090), 278 (11 550), 238 (11300). IR $v_{\max } \mathrm{cm}^{-1}$ : $3400,1740,1640,1580,1500$. MS $m / z$ (rel. int.): $386[\mathrm{M}]^{+}$(7), 249 (75), $221(5), 205(3), 193(20), 189$ (25), $161(12), 137(100), 122(23)$, 105 (11). ${ }^{1} \mathrm{H}$ NMR $\left(\mathrm{CDCl}_{3}\right) \delta 2.58(1 \mathrm{H}, d d, J=14.4,10.2 \mathrm{~Hz}$ $\left.\mathrm{H}-7^{\prime}\right), 3.03\left(1 \mathrm{H}, d d, J=14.4,4.2 \mathrm{~Hz}, \mathrm{H}-7^{\prime}\right), 3.80(3 \mathrm{H}, s, \mathrm{OMe}$ ), 3.82 $(1 \mathrm{H}, m, \mathrm{H}-8), 3.85\left(6 \mathrm{H}, s\right.$, two OMe), $4.22\left(2 \mathrm{H}, m, \mathrm{H}^{-} 9^{\prime}\right), 5.75(1 \mathrm{H}$, br s, OH), $6.06(1 \mathrm{H}, b r s, \mathrm{OH}), 6.58\left(1 \mathrm{H}, d, J=0.9 \mathrm{~Hz}, \mathrm{H}-2^{\prime}\right), 6.62$ $\left(1 \mathrm{H}, d d, J=8.1,0.9 \mathrm{~Hz}, \mathrm{H}-6^{\prime}\right), 6.77(2 \mathrm{H}, \mathrm{s}, \mathrm{H}-2$ and $\mathrm{H}-6), 6.78(1 \mathrm{H}$, $\left.d, J=8.1 \mathrm{~Hz}, \mathrm{H}-5^{\prime}\right), 7.40(1 \mathrm{H}, d, J=1.8 \mathrm{~Hz}, \mathrm{H}-7) .{ }^{13} \mathrm{CNMR}$ $\left(\mathrm{CDCl}_{3}, \mathrm{DEPT}\right): \delta 37.2\left(t, \mathrm{C}-7^{\prime}\right), 39.3\left(d, \mathrm{C}-8^{\prime}\right), 55.8(q, \mathrm{OMe}), 56.3$ ( $q$, two OMe), $69.7\left(t, \mathrm{C}-9^{\prime}\right), 107.4\left(d, \mathrm{C}-2\right.$ and C-6), $111.5\left(d, \mathrm{C}-2^{\prime}\right)$, $114.5\left(d, \mathrm{C}-5^{\prime}\right), 121.0\left(d, \mathrm{C}-6^{\prime}\right), 125.2(s, \mathrm{C}-8), 125.6(s, \mathrm{C}-1), 129.4$ $\left(s, \mathrm{C}-1^{\prime}\right), 137.0(s, \mathrm{C}-4), 137.7(d, \mathrm{C}-7), 144.5\left(s, \mathrm{C}-4^{\prime}\right), 146.6\left(s, \mathrm{C}-3^{\prime}\right)$, $147.2(s, \mathrm{C}-3$ and C-5), $172.7(s, \mathrm{C}-9)$.

\section{REFERENCES}

1. Flora of Taiwan (1975) Vol. 1, p. 538, Epoch, Taiwan.

2. Cheng, Y. S. and Lin, K. C. (1970) Chemistry (Chin.) 28.

3. Cheng, Y. S. and Lin, K. C. (1971) Chemistry (Chin.) 94.

4. Fang, J. M., Jan, S. T. and Cheng, Y. S. (1985) Phytochemistry 24, 1863.

5. Fang, J. M., Jan, S. T. and Cheng, Y. S. (1987) Phytochemistry 26, 853.

6. Fang, J. M., Hsu, K. C. and Cheng, Y. S. (1989) Phytochemistry 28, 1173.

7. Fang, J. M., Hsu, K. C. and Cheng, Y. S. (1989) Phytochemistry 28, 3553.

8. Corrie, J. E. T., Green, G. H., Ritchie, E. and Taylor, W. C. (1970) Aust. J. Chem. 23, 133.

9. Fang, J. M., Wei, K. M. and Cheng, Y. S. (1985) J. Chin. Chem. Soc. (Taipei) 32, 75.

10. Wenkert, E., Gottlieb, H. E., Gottlieb, O. R., Pereira, M. O. S. and Formiga, M. D. (1976) Phytochemistry 15, 1547. 